\title{
Partial plantar fasciectomy for the treatment of plantar fibromatosis
}

\author{
Lucas Plens de Britto Costal ${ }^{(\mathbb{D})}$, Lucas Furtado da Fonsecal ${ }^{\mathbb{D}}$, André Vitor Kerber Cavalcanti Lemos' ${ }^{\mathbb{D}}$, Vinicius Felipe Pereira' ${ }^{(\mathbb{D})}$,

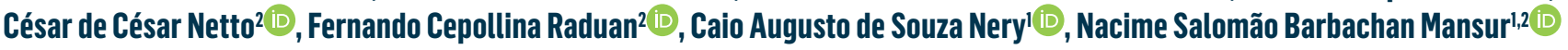 \\ 1. Orthopedics and Traumatology Departament, Universidade Federal de São Paulo (UNIFESP), Paulista School of Medicine, São Paulo, SP, \\ Brazil. \\ 2. Department of Orthopaedics and Rehabilitation, University of Iowa, Iowa City, IA, USA.
}

\begin{abstract}
Objective: We describe a series of patients treated with resection of the affected band. We assessed functional outcome, recurrence rate, and surgical wound complications, comparing these data with the available literature.

Methods: This retrospective study (level of evidence IV) included 14 patients (17 feet) diagnosed with plantar fibromatosis in the medial portion of the fascia that was refractory to conservative treatment. All operations were performed between December 2016 and November 2018. All patients were assessed for symptom improvement and major and minor complications, in addition to recurrence during the study period.
\end{abstract}

Results: Our sample included 9 men and 5 women, whose mean age was 40.6 years (15-63). All of the patients underwent partial fasciectomy of the medial fascial band with margins of at least $2 \mathrm{~cm}$. There was recurrence in 5 of the 17 feet (29\%), but only two required further intervention. Wound dehiscence occurred in 3 patients (17\%), and one of the cases was severe, requiring plastic surgery. Two feet showed signs of injury to the digital branch of the medial plantar nerve.

Conclusion: Partial plantar fasciectomy is an alternative treatment for plantar fibromatosis (Ledderhose's disease). Our results agree with the literature in terms of recurrence and postoperative complications. The moderate rate of complications must be taken into account when indicating this procedure.

Level of Evidence IV; Therapeutic Studies; Case Series.

Keywords: Fibromatosis, plantar; Treatment; Fasciectomy.

\section{Introduction}

Plantar fibromatosis, or Ledderhose's disease, is a locally aggressive, benign disorder characterized by fibroblast proliferation within the plantar aponeurosis and subsequent nodule formation (1). Compared with Dupuytren's disease, an analog of plantar fibromatosis in the hands, little has been published since German physician Georg Ledderhose first reported clinical observations of 50 cases in 1897(2).

The disease is $\operatorname{rare}^{(3)}$, mainly affecting middle aged men, although it has been reported in a nine-month-old infant. One-quarter of the cases are bilateral ${ }^{(4)}$ and the disease is often associated with other forms of hyperproliferative fibromatosis, such as Dupuytren's disease (hands) and Peyronie's disease (penis) ${ }^{(5)}$, as well as other pathologies, such as frozen shoulder, diabetes, and epilepsy ${ }^{(6)}$. Its cause is unknown and probably multifactorial, involving genetic predisposition ${ }^{(6-8)}$. The usual presentation is a slow-growing nodule 5 to $30 \mathrm{~mm}$ in diameter in the medial plantar aponeurosis that does not affect the smooth muscle tissue or the skin and, thus, does not cause contractions ${ }^{(9)}$. Enlargement of the nodule can cause pain in the medial longitudinal arch, especially in the detachment phase ${ }^{(10,11)}$. Clinical management is the mainstay of treatment, including analgesia, corticoid infiltration, and physical therapy ${ }^{(11)}$. If conservative treatment fails, surgery, ie fasciectomy with or without margin and skin coverage (including a graft if necessary), can be considered(12). Surgery with partial or complete resection of the fascia is recommended to reduce the risk of recurrence, as well as thorough wound closure to reduce the risk of dehiscence and painful scarring ${ }^{(11)}$.

We report the surgical outcome of patients who underwent open partial fasciectomy after conservative treatment failed.
Study performed at the Orthopedics and Traumatology Departament, Universidade Federal de São Paulo (UNIFESP), Paulista School of Medicine, São Paulo, SP, Brazil.

Correspondence: Lucas Plens de Britto Costa. Rua Napoleão de Barros, 715, 1ำ andar, Vila Clementino, São Paulo, SP, Brazil. CEP: 04024-002. E-mail: plens.lucas@gmail.com. Conflicts of Interest: none. Source of funding: none. Date received: May 10, 2021. Date accepted: June 15, 2021. Online: August 31, 2021.
How to cite this article: Costa LPB, Fonseca LF, Lemos AVKC, Pereira VF, Cesar Netto C, Raduan FC, et al. Partial plantar fasciectomy for the treatment of plantar fibromatosis. J Foot Ankle. 2021;15(2):124-7. 
Our hypothesis was that open partial fasciectomy would be associated with good functional outcome and low rates of recurrence and wound complications.

\section{Methods \\ Study design}

The study was approved by the institutional research ethics committee and is registered with Plataforma Brasil. This was a retrospective, single-center case series of patients who underwent surgery between December 2016 and November 2018.

\section{Sample}

The sample consisted of patients who underwent open partial fasciectomy after 6 months of conservative treatment (physical therapy, analgesia, and local corticosteroids) failed. Refusal to participate, treatment before or after the stipulated period, and insufficient data to assess the criteria were considered exclusion criteria.

\section{Surgical technique}

Open partial fasciectomy of the affected band was the selected surgical technique. The patient was positioned in horizontal dorsal decubitus, and a curvilinear incision was made under the medial plantar arch, which was carefully dissected to avoid devascularizing the skin. The fascial plane was delineated and the minimum resection margin was $2 \mathrm{~cm}$. The fascia was excised from the adjacent tissue and adequate hemostasis and closure were performed without tension. Sterile dressing was applied for $48 \mathrm{~h}$ after surgery (Figure 1). Load-bearing on the operated limb was prohibited until the wound had completely healed; the stitches were removed within 14 days, provided there were no wound complications.

\section{Outcomes}

Patients were reassessed postoperatively in the first, second, and fourth weeks, as well as in the second, fourth, eighth, and twelfth month. Functional assessment was performed preoperatively and on the final follow-up date. Complications and recurrences were determined at each time point. We evaluated the functional results with the AOFAS Ankle-Hindfoot Evaluation Scale ${ }^{(13)}$.

Mild dehiscence was defined as non-healing 4 weeks after the procedure without the need to provide local coverage, while severe dehiscence was defined as non-healing that required plastic surgery for local coverage. Infection was defined as a discharge of purulent matter from the wound at any time. Any recurrence of the lesion during the study period was included in the analysis ${ }^{(14)}$.

\section{Statistical analysis}

Descriptive statistical analysis (frequency table) was used for non-quantitative variables, while quantitative variables were described as mean and standard deviation. Proportion tests (chi square and adjustments) were performed in Epi Info.
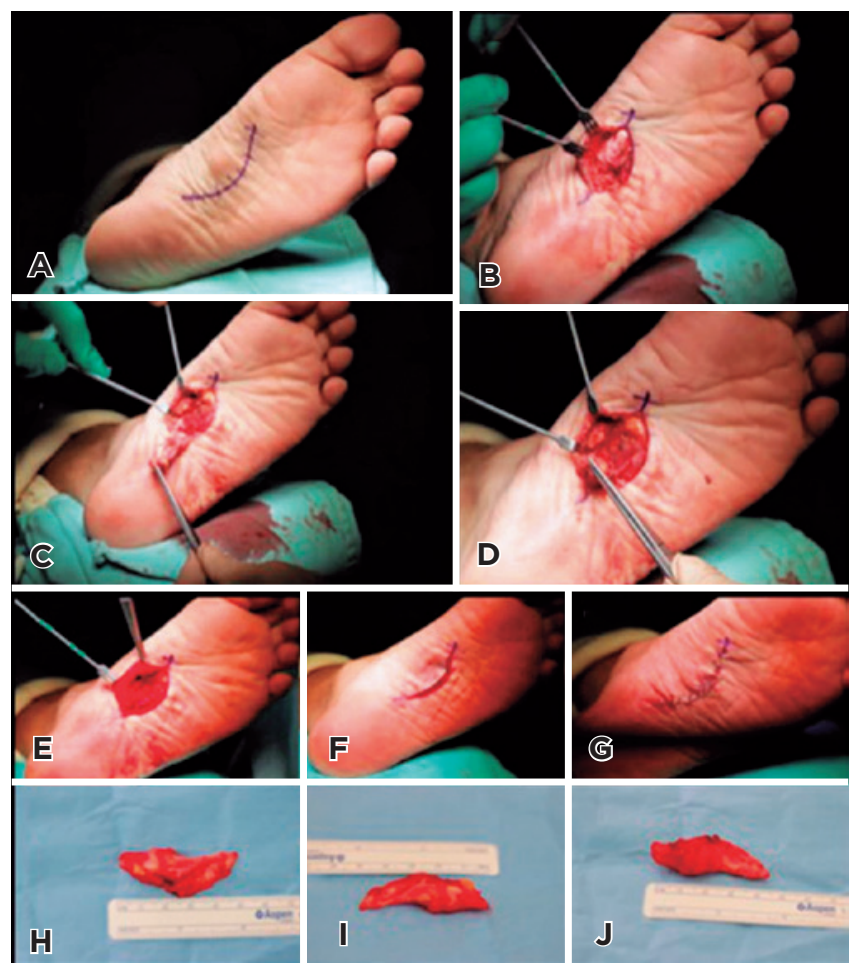

Figure 1. A) The patient is placed in supine position without a cushion. The curvilinear incision lateral to the nodule is demarcated. B) The incision is deepened until a good medial skin flap is formed and the fascia margins can be clearly identified. $C$ and D) The fascia is excised distally to proximally, protecting the adjacent tissue. E) The remaining tissue is inspected, including the free margin of the diseased area and the neurovascular structures. F and G) After thorough hemostasis, the subcutaneous tissue is closed and, finally, the skin is closed with simple stitches. $\mathrm{H}-\mathrm{J}$ ) The dimensions of the excised tissue are determined and documented.

\section{Results}

Fourteen patients diagnosed with plantar fibromatosis in the medial portion of the fascia were included in the study. The sample consisted of 9 men and 5 women, whose mean age was 38.5 years (14-63 years; median 39.85). Since 3 patients had bilateral involvement, a total of 17 feet were treated and evaluated. The right and left foot of one female patient with bilateral involvement were operated on at 14 and 15 years of age, respectively. Apart from this patient, the sample included a 29-year-old man, four women over 39 years of age, and 8 other men over 40 years of age (Table 1).

Wound dehiscence occurred in 3 feet (17\%). Although 2 of the cases were mild and healed adequately after a series of local dressings, the other was severe, requiring plastic surgery for local coverage (Table 2).

There was recurrence in 5 of the 17 feet (29\%) during the study period. However, only two (11.76\%) required surgical re-intervention. 
Table 1. Demographic data of patients with plantar fibromatosis

\begin{tabular}{lcccc} 
Factor & Male & Female & $\begin{array}{c}\text { Mean Age } \\
(\text { Min-Max) }\end{array}$ & $\begin{array}{c}\text { Laterality } \\
\text { (L-R - B) }\end{array}$ \\
\hline Patients $=14$ & 9 & 5 & $\begin{array}{c}40.6 \\
(15-63)\end{array}$ & $11-3-3$ \\
\hline
\end{tabular}

Mean age in years; L: left; R: right; $B$ bilateral.

Table 2. Wound complication rate after surgery

\begin{tabular}{lccccc} 
Factor & $\begin{array}{c}\text { Wound } \\
\text { healed }\end{array}$ & $\begin{array}{c}\text { Wound } \\
\text { dehiscence }\end{array}$ & $\begin{array}{c}\text { Signs of } \\
\text { superficial } \\
\text { infection }\end{array}$ & $\begin{array}{c}\text { Purulent } \\
\text { secretion }\end{array}$ & $\begin{array}{c}\text { Signs } \\
\text { of local } \\
\text { abscess }\end{array}$ \\
\hline $\begin{array}{l}\text { Feet with } \\
\text { PF (n=14) }\end{array}$ & $14(83 \%)$ & $3(17 \%)$ & 0 & 0 & 0 \\
\hline PF: plantar fibromatosis. & & & & \\
\hline
\end{tabular}

Due to the wide margin of excision in one patient, a surgical procedure for local coverage was performed in association with the plastic surgery team. Two feet showed preoperative signs of involvement and damage to the medial plantar nerve. Intraoperatively, it was determined that plantar fibromatosis was involved a branch of this nerve and it was decided to resect it. The patients' mean AOFAS score improved from 48 (37-73) to 84 (68-92) over the study period (Table 3).

\section{Discussion}

This study evaluated the clinical and functional results of partial fasciectomy in patients with Ledderhose's disease and found good postoperative results. AOFAS Ankle-Hindfoot Evaluation Scale scores increased, and the recurrence rate was consistent with the literature. There was a moderate rate of postoperative complications. There were three cases of postoperative wound dehiscence that did not require antibiotic therapy and/or new surgical procedures, in addition to five cases of recurrence, two of which required a new procedure.

After surgical treatment and thorough wound closure, the recurrence rate was $29 \%$, which is consistent with the scant literature $(0-50 \%)^{(15)}$ and superior to local excision of the nodule, which has a recurrence rate of $57-100 \%{ }^{(16)}$.

In our sample there were 3 cases (17\%) of wound dehiscence, including 1 severe case (ie, requiring a new procedure). However, data on surgical wound complications and healing in plantar fibromatosis are scarce. Sammarco and Mangone ${ }^{(15)}$
Table 3. Recurrence rate, pre- and postoperative functional assessment and rate of medial plantar nerve injury

\begin{tabular}{lcccc} 
Factor & Recurrence & $\begin{array}{c}\text { Preoperative } \\
\text { AOFAS score } \\
\text { mean (min.- max.) }\end{array}$ & $\begin{array}{c}\text { Postoperative } \\
\text { AOFAS score } \\
\text { mean (min.- max.) }\end{array}$ & $\begin{array}{c}\text { Medial } \\
\text { plantarnerve } \\
\text { injury }\end{array}$ \\
\hline $\begin{array}{l}\text { Feet with } \\
\text { PF (17) }\end{array}$ & $5(29 \%)$ & $48(37-73)$ & $84(68-92)$ & $2(11 \%)$ \\
\hline PF: & & & \\
\hline
\end{tabular}

PF: plantar fibromatosis

reported 11 cases of dehiscence in 23 operated feet, while Kadir et al. ${ }^{(17)}$ reported 2 cases of pain and 1 case of scar hypersensitivity in 19 operated feet. Moreno et al. ${ }^{(18)}$ reported 18 cases without pain or recurrence in 19 operated feet, with only one case of mild dehiscence.

As the mean functional outcome, the mean AOFAS score for the midfoot increased from 48 (37-73) to 84 (68-92) points. However, only one other study has included this functional outcome $^{(15)}$, in which the AOFAS midfoot score increased from 70 (61-77) to 77 (64-88) points.

One positive point of this study was the varied sample, which included patients of different ages. The researcher who performed the retrospective data collection and analysis did not participate in the consultations, surgeries, satisfaction assessments, or the pre- or postoperative physical examinations. The follow-up period was substantial and there was a considerable sample, considering the disease's rarity and the paucity of articles on surgical results.

Because the surgeries were not performed by the same surgeon, individual skill can be a confounding factor in the results, especially in terms of complications. Despite being a retrospective study with a sample of 17 surgeries, this study has added to the available literature on the treatment of this disease. Moreover, there was no control group or prior sample size/strength calculation. Finally, our method for analyzing surgical wound dehiscence has not been validated.

\section{Conclusion}

Partial plantar fasciectomy is a reasonable alternative treatment for Ledderhose's disease. The results of our study agree with the literature in terms of epidemiology, recurrence, and postoperative complications. The considerable recurrence and reoperation rates must be taken into account when indicating this procedure.

Authors' contributions: Each author contributed individually and significantly to the development of this article: LPBC *(https://orcid.org/OOOO-OOO24955-6822) Data collection, statistical analysis, bibliographic review, formatting of the article, survey of the medical records, statistical analysis, participated in the review process; LFF *(https://orcid.org/O000-0001-6497-833X) Performed the surgeries, data collection, bibliographic review; AVKCL *(https://orcid. org/0000-0001-8974-5815) Performed the surgeries, data collection, bibliographic review; VFP *(https://orcid.org/0000-0002-1005-6089) Performed the surgeries, data collection, bibliographic review; CCN *(https://orcid.org/0000-0001-6037-0685) Data collection, bibliographic review approved the final version; FCR *(https://orcid.org/0000-0002-2922-1929) Performed the surgeries, data collection, bibliographic review clinical examination, approved the final version; CASN *(https://orcid.org/0000-0002-9286-1750) Data collection, bibliographic review approved the final version, interpreted the results of the study; NSBM *(https://orcid.org/0000-0003-1067-727X) Data collection, bibliographic review approved the final version, interpreted the results of the study, conceived and planned the activities that led to the study. All authors read and approved the final manuscript. ${ }^{*}$ ORCID (Open Researcher and Contributor ID) iD. 


\section{References}

1. Carroll P, Henshaw RM, Garwood C, Raspovic K, Kumar D. Plantar Fibromatosis: Pathophysiology, Surgical and Nonsurgical Therapies: An Evidence Based Review. Foot Ankle Spec. 2018;11(2):168-76.

2. Ledderhose, G. Sur pathologic der aponeurote des fusses und der hand. Arch Klin. Chir. 1897;55:694-12.

3. Ledderhose Disease [Internet]. Gaithersburg: National Institutes of Health Genetic and Rare Diseases Information; 2017 [cited 2021 May 8]. Available from: https://rarediseases.info.nih.gov/diseases/ 6873/ledderhose-disease

4. Neagu TP, Sinescu RD, Enache V, Achim SC, Tigliș M, Mirea LE. Metastatic high-grade myxofibrosarcoma: review of a clinical case. Rom J Morphol Embryol. 2017;58(2):603-9.

5. Mohede DCJ, Riesmeijer SA, de Jong IJ, Werker PMN, van Driel MF. Prevalence of Peyronie and Ledderhose Diseases in a Series of 730 Patients with Dupuytren Disease. Plast Reconstr Surg. 2020;145(4):978-84.

6. Akdag O, Yildiran G, Karamese M, Tosun Z. Dupuytren-Like Contracture of the Foot: Ledderhose Disease. Surg J (N Y). 2016; 2(3):e102-e4

7. Kim SK, loannidis JPA, Ahmed MA, Avins AL, Kleimeyer JP, Fredericson M, et al. Two Genetic Variants Associated with Plantar Fascial Disorders. Int J Sports Med. 2018;39(4):314-21.

8. Grenfell S, Borg M. Radiotherapy in fascial fibromatosis: a case series, literature review and considerations for treatment of earlystage disease. J Med Imaging Radiat Oncol. 2014;58(5):641-7.

9. Young JR, Sternbach S, Willinger M, Hutchinson ID, Rosenbaum AJ. The etiology, evaluation, and management of plantar fibromatosis. Orthop Res Rev. 2019;11:1-7.
10. Yasui Y, Takao M, Miyamoto W, Matsushita T. Plantar fibromatosis with flexion contracture and valgus deformity of the great toe. J Orthop Sci. 2016;21(3):395-8.

11. Veith NT, Tschernig T, Histing T, Madry $\mathrm{H}$. Plantar fibromatosistopical review. Foot Ankle Int. 2013;34(12):1742-6.

12. Yin MC, Ye J, Yao M, Cui XJ, Xia Y, Shen QX, et al. Is extracorporea shock wave therapy clinical efficacy for relief of chronic, recalcitrant plantar fasciitis? A systematic review and meta-analysis of randomized placebo or active-treatment controlled trials. Arch Phys Med Rehabil. 2014;95(8):1585-93.

13. Rodrigues RC, Masiero D, Mizusaki JM, Imoto AM, Peccin MS Cohen $\mathrm{M}$, et al. Translation, cultural adaptation and validity of the "American Orthopaedic Foot and Ankle Society (AOFAS) Ankle-Hindfoot Scale. Acta Ortop Bras. 2008;16(2):107-111.

14. Mansur NSB, Lemos AVKC, Baumfeld DS, Baumfeld TS, Prado MP, Raduan FC, et al. Medial and lateral combined ligament arthroscopic repair for multidirectional ankle instability. Foot Ankle Orthop. 2021;6(1):1-8

15. Sammarco GJ, Mangone PG. Classification and treatment of plantar fibromatosis. Foot Ankle Int. 2000;21(7):563-9.

16. Knobloch K, Vogt PM. High-energy focussed extracorporeal shockwave therapy reduces pain in plantar fibromatosis (Ledderhose's disease) BMC Res Notes. 2012;5:542.

17. Kadir HKA, Chandrasekar CR. Partial fasciectomy is a usefu treatment option for symptomatic plantar fibromatosis. Foot (Edinb). 2017;31:31-4

18. Moreno MVMG, Henkes GC, Costa FA, Gouvêa RS, Schiper L. Surgery treatment of Ledderhose disease with the wide ressection technique of the plantar fascia. Rev ABTPé. 2007;1(1):39-43. 